\title{
Consistent operator semigroups and their interpolation
}

\author{
A.F.M. ter Elst and J. Rehberg
}

\begin{abstract}
Under a mild regularity condition we prove that the generator of the interpolation of two $C_{0}$-semigroups is the interpolation of the two generators.
\end{abstract}

\section{Introduction}

Interpolation is a main tool in parabolic differential equations and in particular in semigroup theory, see [BB], [Tri, Section 1.13] and [Lun, Chapter 2]. Frequently interpolation is done between two $L^{p}$-spaces or between a Banach space and the domain of a power of the generator of a semigroup. The aim of this paper is to consider abstractly interpolation of continuous semigroups, from the viewpoint of category theory. In one of the main theorems of this paper, Theorem 3.10, we show that the generator of the interpolation of two $C_{0}$-semigroups is the interpolation of the two generators. As a corollary this gives the following theorem for complex interpolation.

Theorem 1.1. Let $(X, \mathcal{A}, \mu)$ be a $\sigma$-finite measure space and let $p_{0}, p_{1} \in[1, \infty)$. Let $S^{\left(p_{0}\right)}$ and $S^{\left(p_{1}\right)}$ be bounded consistent $C_{0}$-semigroups in $L^{p_{0}}$ and $L^{p_{1}}$ with generators $-A_{p_{0}}$ and $-A_{p_{1}}$, respectively. Let $\theta \in[0,1]$ and let $p \in[1, \infty)$ be such that $\frac{1}{p}=\frac{1-\theta}{p_{0}}+\frac{\theta}{p_{1}}$. Let $S^{(p)}$ be the $C_{0}$-semigroup on $L^{p}$ which is consistent with $S^{\left(p_{0}\right)}$. Let $-A_{p}$ be the generator of $S^{(p)}$. Then

$$
\left[D\left(A_{p_{0}}\right), D\left(A_{p_{1}}\right)\right]_{\theta}=D\left(A_{p}\right) .
$$

In order to illustrate the abstract setting of the paper we give an example in non-linear parabolic equations, where the appropriate interpolation is not between two $L^{p}$-spaces or between a Banach space and a power of a semigroup generator. Consider the quasilinear initial boundary value problem

$$
u^{\prime}-\nabla \cdot \phi(u) \nabla u=|\nabla u|^{2},\left.\quad \nu \cdot \nabla u\right|_{\partial \Omega}=f \neq 0, \quad u(0)=0 .
$$

on a three-dimensional (possibly nonsmooth) domain $\Omega$, where $u \in L^{s}((0, T) ; X)$. We wish to find a suitable Banach space $X$ for the treatment of this initial boundary value

Mathematics Subject Classification. 46B70, 46M35, 47D06.

Keywords. Semigroups, interpolation. 
problem. First, if the boundary conditions are replaced by a Dirichlet condition, then $L^{p}$-spaces are an adequate choice for $X$ in order to treat (1.1), see [MERS] and [HR]. In view of the inhomogeneous Neumann conditions the $L^{p}$-spaces are not suitable (cf. [Cia, Section 1.2] [GGZ, Subsection II.2.2]). Secondly, at a first glance, the choice $X=W^{-1,2}(\Omega)$, which is the dual of $W^{1,2}(\Omega)$, seems to be adequate for the problem (1.1), as in the linear case, compare [Lio, Section 3.3]. With this choice, however, for every fixed $t$ the function $u(t, \cdot)$ is then in general an element of $W^{1,2}(\Omega)$ and hence $|\nabla u(t, \cdot)|^{2}$ is then an element of $L^{1}(\Omega)$ and fails to be an element of $X$, as required by the differential equation in (1.1). Thirdly, replacing $W^{-1,2}(\Omega)$ by the smaller space $W^{-1, q}(\Omega)$, with $q$ larger than the spatial dimension 3, and under the condition that $-\Delta+I: W^{1, q}(\Omega) \rightarrow W^{-1, q}(\Omega)$ is a topological isomorphism (cf. [Zan] and [HHKRZ]) one could now guess that $X:=W^{-1, q}(\Omega)$ is a good space to treat (1.1). Indeed, one could then reflect the inhomogeneous Neumann condition adequately. Moreover, since $u(t, \cdot) \in \operatorname{dom}(\Delta)=W^{1, q}(\Omega)$ one deduces that $|\nabla u(t, \cdot)|^{2} \in$ $L^{q / 2}(\Omega) \subset W^{-1, q}(\Omega)$, where we used that $q$ is larger than the space dimension 3 . This means that $|\nabla u(t, \cdot)|^{2} \in X$ for every element $u(t, \cdot)$ in the domain of the elliptic operator and each $t>0$. But, unfortunately, the theory for quasi-linear parabolic equations requires more: for elements from an interpolation space between the Banach space and the domain of the elliptic operator the right hand side $|\nabla u|^{2}$ of the differential equation in (1.1) has to be well-behaved in order to assure at least (local in time) existence and uniqueness, see for example [Lun, Chapter 7]. But for elements $v$ of an interpolation space between $W^{-1, q}(\Omega)$ and $W^{1, q}(\Omega)$, the gradient $\nabla v$ cannot be expected to be a function in general, and $|\nabla v|^{2}$ cannot be defined properly for such elements (for the required interpolation arguments see GGKR).

In order to get out of this dilemma it turns out that, quite in coincidence with the concept in [CS], that one should take for $X$ a Banach space which fulfils the following three properties.

(II) $|\nabla \psi|^{2} \in X$ for all $\psi \in W^{1, q}$ and

(III) $-\Delta$ generates on $X$ a (suitably regular) semigroup.

In [HDR, Section 7] a comprehensive treatment for (1.1) is given in a (dual) Bessel function space $H^{-\tau, q}=\left[L^{q}, W^{-1, q}\right]_{\theta}$ with $\tau \in\left(\frac{3}{q}, 1\right)$. But in the meanwhile it turned out that passing to spaces $\left[L^{p}, W^{-1, q}\right]_{\theta}$ with $p \neq q$ gives the theory more flexibility - and sharper results. In particular, $p=\frac{q}{2}$ is of special interest, see [BMNR. The reason of this are the better multiplier properties in the expression $\nabla \cdot \phi \nabla$ in the dependence of $\phi$. Note that then the space $X=\left[L^{p}, W^{-1, q}\right]_{\theta}$ is generally not an interpolation space between the general Banach space $W^{-1, q}$ and the corresponding domain of the Laplacian (as a reference operator) on $W^{-1, q}$. It turns out that indeed Properties (I), (II) and (III) are satisfied when taking $X=\left[L^{p}, W^{-1, q}\right]_{\theta}$ and $p$ is suitably chosen. In particular, the semigroup generator property for the operators $-\nabla \cdot \phi \nabla$ on $X=\left[L^{p}, W^{-1, q}\right]_{\theta}$ is expected to follow from the 
generator property on both $L^{p}$ and $W^{-1, q}$ by interpolation. Indeed, this is fairly clear in case of analytic semigroups, but not in general. This is one of our motivations to investigate interpolation properties for suitable operator semigroups in a more general context.

Since one is usually interested in a concrete equation, one must first know that the two semigroups act consistently on the involved Banach spaces. In Section 2 we characterise consistency of semigroups in terms of their resolvents and we obtain a useful expression for the intersection of the domain of the generators. In Section 3 we consider interpolation functors and prove loosely speaking that semigroup generators and interpolation functors commute. In the last section we give a couple of examples in $L^{p}$-spaces and distribution spaces for consistent semigroups.

\section{Consistency of operator semigroups}

In this section we show that two $C_{0}$-semigroups are consistent if and only if the resolvents of the generators are consistent for large $\lambda>0$. We start we the definition of consistent operators.

Definition 2.1. Let $X$ and $Y$ be two vector spaces. Let $T_{0}: D\left(T_{0}\right) \rightarrow Y$ and $T_{1}: D\left(T_{1}\right) \rightarrow$ $Y$ be two (linear) operators with domains $D\left(T_{0}\right) \subset X$ and $D\left(T_{1}\right) \subset X$. Then the operators $T_{0}$ and $T_{1}$ are called consistent if $T_{0} x=T_{1} x$ for all $x \in D\left(T_{0}\right) \cap D\left(T_{1}\right)$. Let $X_{0}$ and $X_{1}$ be two Banach spaces which are embedded in a vector space $X$. Let $S^{(0)}$ and $S^{(1)}$ be semigroups in $X_{0}$ and $X_{1}$, respectively. Then the semigroups $S^{(0)}$ and $S^{(1)}$ are called consistent if $S_{t}^{(0)}$ and $S_{t}^{(1)}$ are consistent for all $t>0$.

The following easy lemma gives a sufficient condition for two bounded operators to be consistent.

Lemma 2.2. Let $\left(X_{0}, X_{1}\right)$ be an interpolation couple of Banach spaces. Let $T_{0}$ and $T_{1}$ be bounded operators in $X_{0}$ and $X_{1}$, respectively. Let $D \subset X_{0} \cap X_{1}$ and suppose that $D$ is dense in $X_{0} \cap X_{1}$. Further, suppose that $T_{0} x=T_{1} x$ for all $x \in D$. Then $T_{0}$ and $T_{1}$ are consistent.

The boundedness condition on the semigroups in the sequel is just for convenience.

Lemma 2.3. Let $\left(X_{0}, X_{1}\right)$ be an interpolation couple of Banach spaces. Let $S^{(0)}$ and $S^{(1)}$ be bounded $C_{0}$-semigroups in $X_{0}$ and $X_{1}$ with generators $-A_{0}$ and $-A_{1}$, respectively. Then the following are equivalent.

(i) The semigroups $S^{(0)}$ and $S^{(1)}$ are consistent.

(ii) For all $\lambda>0$ the resolvent operators $\left(A_{0}+\lambda I\right)^{-1}$ and $\left(A_{1}+\lambda I\right)^{-1}$ are consistent.

Proof. (i) $\Rightarrow($ ii $)$. Let $\lambda>0$ and $x \in X_{0} \cap X_{1}$. Then

$$
\left(A_{0}+\lambda I\right)^{-1} x=\int_{0}^{\infty} e^{-\lambda t} S_{t}^{(0)} x d t=\int_{0}^{\infty} e^{-\lambda t} S_{t}^{(1)} x d t=\left(A_{1}+\lambda I\right)^{-1} x
$$


(ii) $\Rightarrow$ (i) . Let $\lambda>0$ and $x \in X_{0} \cap X_{1}$. Then it follows by induction to $n$ that $\left(A_{0}+\lambda I\right)^{-n} x=\left(A_{1}+\lambda I\right)^{-n} x$ for all $n \in \mathbb{N}$. Now let $t>0$ and $x \in X_{0} \cap X_{1}$. Then the Euler formula gives

$$
S_{t}^{(0)} x=\lim _{n \rightarrow \infty}\left(A_{0}+\frac{t}{n} I\right)^{-n} x=\lim _{n \rightarrow \infty}\left(A_{1}+\frac{t}{n} I\right)^{-n} x=S_{t}^{(1)} x,
$$

as required.

Remark 2.4. In Are Proposition 2.2 the following is proved: the set $\mathcal{U}$ of all $\lambda$ for which $\left(A_{0}+\lambda I\right)^{-1}$ and $\left(A_{1}+\lambda I\right)^{-1}$ are consistent, is open and closed in $\rho\left(-A_{0}\right) \cap \rho\left(-A_{1}\right)$. From this it easily follows that if $\rho\left(-A_{0}\right)=\rho\left(-A_{1}\right)$ and this set is connected, then the consistency of $\left(A_{0}+\lambda_{0} I\right)^{-1}$ and $\left(A_{1}+\lambda_{0} I\right)^{-1}$ for only one $\lambda_{0}$ implies the consistency of all resolvent operators.

If the equivalent conditions in Lemma 2.3 are valid, then it is possible that there exists a $\lambda \in \rho\left(-A_{0}\right) \cap \rho\left(-A_{1}\right)$ such that the resolvents $\left(A_{0}+\lambda I\right)^{-1}$ and $\left(A_{1}+\lambda I\right)^{-1}$ are not consistent. An example has been given in Are Section 3.

Proposition 2.5. Let $\left(X_{0}, X_{1}\right)$ be an interpolation couple of Banach spaces. Let $S^{(0)}$ and $S^{(1)}$ be bounded consistent $C_{0}$-semigroups in $X_{0}$ and $X_{1}$ with generators $-A_{0}$ and $-A_{1}$, respectively. Then one has the following.

(a) The generators $A_{0}$ and $A_{1}$ are consistent.

(b) $D\left(A_{0}\right) \cap D\left(A_{1}\right)=\left\{x \in D\left(A_{0}\right) \cap X_{1}: A_{0} x \in X_{1}\right\}=\left(A_{0}+I\right)^{-1}\left(X_{0} \cap X_{1}\right)$.

Proof. (a). Let $x \in D\left(A_{0}\right) \cap D\left(A_{1}\right)$ and $F \in\left(X_{0}+X_{1}\right)^{\prime}$. Then

$$
F\left(A_{0} x\right)=\lim _{t \downarrow 0} \frac{1}{t} F\left(\left(I-S^{(0)}\right) x\right)=\lim _{t \downarrow 0} \frac{1}{t} F\left(\left(I-S^{(1)}\right) x\right)=F\left(A_{1} x\right) .
$$

Hence $A_{0} x=A_{1} x$.

(b). Let $x \in D\left(A_{0}\right) \cap D\left(A_{1}\right)$. Then it follows from Statement (a) that $A_{0} x=A_{1} x \in X_{1}$. So $D\left(A_{0}\right) \cap D\left(A_{1}\right) \subset\left\{x \in D\left(A_{0}\right) \cap X_{1}: A_{0} x \in X_{1}\right\}$. Conversely, suppose $x \in D\left(A_{0}\right) \cap X_{1}$ and $A_{0} x \in X_{1}$. Let $t>0$. Then for all $F \in\left(X_{0}+X_{1}\right)^{\prime}$ one deduces that

$$
\begin{aligned}
F\left(\left(I-S_{t}^{(1)}\right) x\right) & =F\left(\left(I-S_{t}^{(0)}\right) x\right)=F\left(\int_{0}^{t} S_{s}^{(0)} A_{0} x d s\right)=\int_{0}^{t} F\left(S_{s}^{(0)} A_{0} x\right) d s \\
& =\int_{0}^{t} F\left(S_{s}^{(1)} A_{0} x\right) d s=F\left(\int_{0}^{t} S_{s}^{(1)} A_{0} x d s\right) .
\end{aligned}
$$

So

in $X_{1}$. Hence

$$
\frac{1}{t}\left(I-S_{t}^{(1)}\right) x=\frac{1}{t} \int_{0}^{t} S_{s}^{(1)} A_{0} x d s
$$

$$
\lim _{t \downarrow 0} \frac{1}{t}\left(I-S_{t}^{(1)}\right) x=A_{0} x
$$

in $X_{1}$. Therefore $x \in D\left(A_{1}\right)$. This proves the first equality in Statement (b).

Next, let $x \in D\left(A_{0}\right) \cap D\left(A_{1}\right)$. Then $\left(A_{0}+I\right) x=\left(A_{1}+I\right) x \in X_{0} \cap X_{1}$ again by Statement (a), So $x \in\left(A_{0}+I\right)^{-1}\left(X_{0} \cap X_{1}\right)$. Conversely, let $x \in X_{0} \cap X_{1}$. Then obviously $\left(A_{0}+I\right)^{-1} u \in D\left(A_{0}\right)$. Since $\left(A_{0}+I\right)^{-1}$ and $\left(A_{1}+I\right)^{-1}$ are consistent by Lemma 2.3, it follows that $\left(A_{0}+I\right)^{-1} x=\left(A_{1}+I\right)^{-1} x \in D\left(A_{1}\right)$. So $\left(A_{0}+I\right)^{-1} x \in D\left(A_{0}\right) \cap D\left(A_{1}\right)$. 


\section{Interpolation of consistent operator semigroups}

In this section we consider interpolation of semigroups and their generators. In all what follows, we adopt the terminology of [Tri] Section 1.2, with minor modifications.

Let $\left(X_{0}, X_{1}\right)$ and $\left(Y_{0}, Y_{1}\right)$ be two interpolation couples of Banach spaces. Recall from [Tri Subsection 1.2.2 that $L\left(\left(X_{0}, X_{1}\right),\left(Y_{0}, Y_{1}\right)\right)$ denotes the vector space of all linear maps $T: X_{0}+X_{1} \rightarrow Y_{0}+Y_{1}$ such that $\left.T\right|_{X_{0}} \in \mathcal{L}\left(X_{0}, Y_{0}\right)$ and $\left.T\right|_{X_{1}} \in \mathcal{L}\left(X_{1}, Y_{1}\right)$. Clearly the operators $\left.T\right|_{X_{0}}$ and $\left.T\right|_{X_{1}}$ are consistent for all $T \in L\left(\left(X_{0}, X_{1}\right),\left(Y_{0}, Y_{1}\right)\right)$. There is a converse.

Lemma 3.1. Let $\left(X_{0}, X_{1}\right)$ and $\left(Y_{0}, Y_{1}\right)$ be two interpolation couples of Banach spaces, $T_{0} \in \mathcal{L}\left(X_{0}, Y_{0}\right)$ and $T_{1} \in \mathcal{L}\left(X_{1}, Y_{1}\right)$. Suppose that $T_{0}$ and $T_{1}$ are consistent. Then there exists a unique $T \in L\left(\left(X_{0}, X_{1}\right),\left(Y_{0}, Y_{1}\right)\right)$ such that $\left.T\right|_{X_{0}}=T_{0}$ and $\left.T\right|_{X_{1}}=T_{1}$.

Moreover, the operator $T$ is continuous from $X_{0}+X_{1}$ into $Y_{0}+Y_{1}$ and $\|T\|_{X_{0}+X_{1} \rightarrow Y_{0}+Y_{1}} \leq$ $\left\|T_{0}\right\|_{X_{0} \rightarrow Y_{0}} \vee\left\|T_{1}\right\|_{X_{1} \rightarrow Y_{1}}$.

Proof. The first part is easy and $T \in L\left(\left(X_{0}, X_{1}\right),\left(Y_{0}, Y_{1}\right)\right)$ is given by $T\left(x_{0}+x_{1}\right)=$ $T_{0} x_{0}+T_{1} x_{1}$ for all $x_{0} \in X_{0}$ and $x_{1} \in X_{1}$. Here we use that $T_{0}$ and $T_{1}$ are consistent.

Next, let $x \in X_{0}+X_{1}$. Let $x_{0} \in X_{0}$ and $x_{1} \in X_{1}$ be such that $x=x_{0}+x_{1}$. Then

$$
\begin{aligned}
\|T x\|_{X_{0}+X_{1}} & \leq\left\|T_{0} x_{0}\right\|_{X_{0}}+\left\|T_{1} x_{1}\right\|_{X_{1}} \\
& \leq\left(\left\|T_{0}\right\|_{X_{0} \rightarrow Y_{0}} \vee\left\|T_{1}\right\|_{X_{1} \rightarrow Y_{1}}\right)\left(\left\|x_{0}\right\|_{X_{0}}+\left\|x_{1}\right\|_{X_{1}}\right) .
\end{aligned}
$$

So $\|T x\|_{Y_{0}+Y_{1}} \leq\left(\left\|T_{0}\right\|_{X_{0} \rightarrow Y_{0}} \vee\left\|T_{1}\right\|_{X_{1} \rightarrow Y_{1}}\right)\|x\|_{X_{0}+X_{1}}$. This proves the last assertion.

Let $\left(X_{0}, X_{1}\right)$ and $\left(Y_{0}, Y_{1}\right)$ be two interpolation couples of Banach spaces. We provide $L\left(\left(X_{0}, X_{1}\right),\left(Y_{0}, Y_{1}\right)\right)$ with the norm

$$
\|T\|_{L\left(\left(X_{0}, X_{1}\right),\left(Y_{0}, Y_{1}\right)\right)}=\left\|\left.T\right|_{X_{0}}\right\|_{X_{0} \rightarrow Y_{0}} \vee\left\|\left.T\right|_{X_{1}}\right\|_{X_{1} \rightarrow Y_{1}} .
$$

Then $L\left(\left(X_{0}, X_{1}\right),\left(Y_{0}, Y_{1}\right)\right)$ is a Banach space. For the concept of interpolation functor we refer to [Tri] Subsection 1.2.2. If $\mathcal{F}$ is an interpolation functor and $T \in L\left(\left(X_{0}, X_{1}\right),\left(Y_{0}, Y_{1}\right)\right)$, then we denote by $T^{\mathcal{F}}: \mathcal{F}\left(X_{0}, X_{1}\right) \rightarrow \mathcal{F}\left(Y_{0}, Y_{1}\right)$ the restriction of $T$ to $\mathcal{F}\left(X_{0}, X_{1}\right)$. Note that $T^{\mathcal{F}}$ is a bounded operator. Alternatively, since we are interested in consistent operators, we also introduce another notation. Let $T_{0} \in \mathcal{L}\left(X_{0}, Y_{0}\right)$ and $T_{1} \in \mathcal{L}\left(X_{1}, Y_{1}\right)$. Suppose that $T_{0}$ and $T_{1}$ are consistent. By Lemma 3.1 there exists a unique $T \in L\left(\left(X_{0}, X_{1}\right),\left(Y_{0}, Y_{1}\right)\right)$ such that $\left.T\right|_{X_{0}}=T_{0}$ and $\left.T\right|_{X_{1}}=T_{1}$. Then we define

$$
\mathcal{F}\left(T_{0}, T_{1}\right)=T^{\mathcal{F}}
$$

So $\mathcal{F}\left(T_{0}, T_{1}\right)$ is a bounded operator from $\mathcal{F}\left(X_{0}, X_{1}\right)$ into $\mathcal{F}\left(Y_{0}, Y_{1}\right)$. Since $T_{0}, T_{1}$ and $\mathcal{F}\left(T_{0}, T_{1}\right)=T^{\mathcal{F}}$ are all three restrictions of the same operator $T$ on $X_{0}+X_{1}$, it is obvious that the three operators $T_{0}, T_{1}$ and $\mathcal{F}\left(T_{0}, T_{1}\right)=T^{\mathcal{F}}$ are pairwise consistent.

Lemma 3.2. Let $\left(X_{0}, X_{1}\right)$ and $\left(Y_{0}, Y_{1}\right)$ be two interpolation couples of Banach spaces and $\mathcal{F}$ an interpolation functor. Then there exists an $M>0$ such that

$$
\left\|T^{\mathcal{F}}\right\|_{\mathcal{F}\left(X_{0}, X_{1}\right) \rightarrow \mathcal{F}\left(Y_{0}, Y_{1}\right)} \leq M\|T\|_{L\left(\left(X_{0}, X_{1}\right),\left(Y_{0}, Y_{1}\right)\right)}
$$

for all $T \in L\left(\left(X_{0}, X_{1}\right),\left(Y_{0}, Y_{1}\right)\right)$. 
Proof. The operator $T \mapsto T^{\mathcal{F}}$ from the Banach space $L\left(\left(X_{0}, X_{1}\right),\left(Y_{0}, Y_{1}\right)\right)$ into the Banach space $\mathcal{L}\left(\mathcal{F}\left(X_{0}, X_{1}\right), \mathcal{F}\left(Y_{0}, Y_{1}\right)\right)$ has a closed graph.

In several contexts dual semigroups are of interest, see the papers Ama, AE]. Therefore it makes sense to establish a connection between consistency of operators and consistency of their adjoints.

Proposition 3.3. Let $\left(X_{0}, X_{1}\right)$ and $\left(Y_{0}, Y_{1}\right)$ be two interpolation couples of Banach spaces, $T_{0} \in \mathcal{L}\left(X_{0}, Y_{0}\right)$ and $T_{1} \in \mathcal{L}\left(X_{1}, Y_{1}\right)$. Suppose that $T_{0}$ and $T_{1}$ are consistent. Let $T \in$ $L\left(\left(X_{0}, X_{1}\right),\left(Y_{0}, Y_{1}\right)\right)$ be such that $\left.T\right|_{X_{0}}=T_{0}$ and $\left.T\right|_{X_{1}}=T_{1}$. Then one has the following.

(a) $\quad T^{\prime}=\left.T_{0}^{\prime}\right|_{\left(Y_{0}+Y_{1}\right)^{\prime}}=\left.T_{1}^{\prime}\right|_{\left(Y_{0}+Y_{1}\right)^{\prime}}$.

(b) If $Y_{0} \cap Y_{1}$ is dense in both spaces $Y_{0}$ and $Y_{1}$, then

$$
\left(Y_{0}+Y_{1}\right)^{\prime}=Y_{0}^{\prime} \cap Y_{1}^{\prime}
$$

and, consequently,

$$
T^{\prime}=\left.T_{0}^{\prime}\right|_{Y_{0}^{\prime} \cap Y_{1}^{\prime}}=\left.T_{1}^{\prime}\right|_{Y_{0}^{\prime} \cap Y_{1}^{\prime}}
$$

Proof. (a). Clearly the adjoint $T^{\prime}$ of $T$ is a continuous operator from $\left(Y_{0}+Y_{1}\right)^{\prime}$ into $\left(X_{0}+X_{1}\right)^{\prime}$. Let $f \in\left(Y_{0}+Y_{1}\right)^{\prime} \subset Y_{0}^{\prime}$ and $x \in X_{0} \subset X_{0}+X_{1}$. Then

$$
\left\langle T_{0}^{\prime} f, x\right\rangle_{X_{0}^{\prime} \times X_{0}}=\left\langle f, T_{0} x\right\rangle_{Y_{0}^{\prime} \times Y_{0}}=\langle f, T x\rangle_{\left(Y_{0}+Y_{1}\right)^{\prime} \times\left(Y_{0}+Y_{1}\right)}=\left\langle T^{\prime} f, x\right\rangle_{\left(X_{0}+X_{1}\right)^{\prime} \times\left(X_{0}+X_{1}\right)} .
$$

The second equality is proved analogously.

(b)]. Under the density condition, the equality (3.1) is well-known, cf. [BL76] Theorem 2.7.1. Then (3.2) follows from (a).

Definition 3.4. We say that an interpolation functor $\mathcal{F}$ has Property (d) (for dense) if for every interpolation couple $\left(X_{0}, X_{1}\right)$ the subspace $X_{0} \cap X_{1}$ is dense in the interpolation space $\mathcal{F}\left(X_{0}, X_{1}\right)$.

Example 3.5. The complex interpolation has Property (d). With exception of the limit values also the real interpolation has Property (d). For complex and real interpolation, see [Tri] Subsections 1.9.3 and 1.6.2.

Example 3.6. The real interpolation with parameters the limit values does not have Property (d), see [Tri] Remark 1.18.3.5.

The next lemma is easy to prove.

Lemma 3.7. Let $\left(X_{0}, X_{1}\right)$ and $\left(Y_{0}, Y_{1}\right)$ be two interpolation couples of Banach spaces and $\mathcal{F}$ an interpolation functor which has Property $(d)$. Let $T_{0} \in \mathcal{L}\left(X_{0}, Y_{0}\right), T_{1} \in \mathcal{L}\left(X_{1}, Y_{1}\right)$ and suppose that $T_{0}$ and $T_{1}$ are consistent. Then $\mathcal{F}\left(T_{0}, T_{1}\right)$ is the unique extension of the operator $\left.T\right|_{X_{0} \cap X_{1}}: X_{0} \cap X_{1} \rightarrow Y_{0} \cap Y_{1}$ which is continuous from the space $\mathcal{F}\left(X_{0}, X_{1}\right)$ into the space $\mathcal{F}\left(Y_{0}, Y_{1}\right)$. 
Next we consider a functor on consistent semigroups.

Proposition 3.8. Let $\mathcal{F}$ be an interpolation functor. Let $\left(X_{0}, X_{1}\right)$ be an interpolation couple of Banach spaces. Let $S^{(0)}$ and $S^{(1)}$ be consistent semigroups in $X_{0}$ and $X_{1}$ respectively. Then one has the following.

(a) The family $\left(\mathcal{F}\left(S_{t}^{(0)}, S_{t}^{(1)}\right)\right)_{t>0}$ on $\mathcal{F}\left(X_{0}, X_{1}\right)$ is a semigroup which is consistent with both $S^{(0)}$ and $S^{(1)}$.

(b) If both $S^{(0)}$ and $S^{(1)}$ are bounded semigroups, then the semigroup $\left(\mathcal{F}\left(S_{t}^{(0)}, S_{t}^{(1)}\right)\right)_{t>0}$ is also bounded.

(c) Suppose in addition that $S^{(0)}$ and $S^{(1)}$ are $C_{0}$-semigroups and that the interpolation functor $\mathcal{F}$ has Property (d). Then the semigroup $\left(\mathcal{F}\left(S_{t}^{(0)}, S_{t}^{(1)}\right)\right)_{t>0}$ is a $C_{0^{-}}$ semigroup.

Proof. (a). This is straightforward.

(b). This follows from Lemmas 3.1 and 3.2 .

(c)]. Without loss of generality we may assume that both $S^{(0)}$ and $S^{(1)}$ are bounded semigroups. For all $t>0$ write $S_{t}^{\mathcal{F}}=\mathcal{F}\left(S_{t}^{(0)}, S_{t}^{(1)}\right)$. Then also $\left(S_{t}^{\mathcal{F}}\right)_{t>0}$ is a bounded semigroup by Statement (b).

Since $\mathcal{F}\left(X_{0}, X_{1}\right)$ is an intermediate space for the interpolation couple $\left(X_{0}, X_{1}\right)$, there exists a $c>0$ such that $\|x\|_{\mathcal{F}\left(X_{0}, X_{1}\right)} \leq c\|x\|_{X_{0} \cap X_{1}}$ for all $x \in X_{0} \cap X_{1}$. Let $x \in X_{0} \cap X_{1}$ and $t>0$. Then

$$
\left\|S_{t}^{\mathcal{F}} x-x\right\|_{\mathcal{F}\left(X_{0}, X_{1}\right)} \leq c\left\|S_{t}^{\mathcal{F}} x-x\right\|_{X_{0} \cap X_{1}}=c\left(\left\|S_{t}^{(0)} x-x\right\|_{X_{0}}+\left\|S_{t}^{(1)} x-x\right\|_{X_{1}}\right) .
$$

Hence $\lim _{t \downarrow 0}\left\|S_{t}^{\mathcal{F}} x-x\right\|_{\mathcal{F}\left(X_{0}, X_{1}\right)}=0$ and $\lim _{t \downarrow 0} S_{t}^{\mathcal{F}} x=x$ in $\mathcal{F}\left(X_{0}, X_{1}\right)$.

Finally, $X_{0} \cap X_{1}$ is dense in $\mathcal{F}\left(X_{0}, X_{1}\right)$ since the interpolation functor has Property $(\mathrm{d})$. So $\lim _{t \downarrow 0} S_{t}^{\mathcal{F}} x=x$ in $\mathcal{F}\left(X_{0}, X_{1}\right)$ for all $x \in \mathcal{F}\left(X_{0}, X_{1}\right)$.

We wish to determine the generator of the semigroup $S^{\mathcal{F}}$. We need a lemma.

Lemma 3.9. Let $\mathcal{F}$ be an interpolation functor which has Property (d). Let $\left(X_{0}, X_{1}\right)$ be an interpolation couple of Banach spaces. Further, let $S^{(0)}$ and $S^{(1)}$ be consistent $C_{0}$-semigroups in $X_{0}$ and $X_{1}$ with generators $-A_{0}$ and $-A_{1}$, respectively. Let $S^{\mathcal{F}}=$ $\left(\mathcal{F}\left(S_{t}^{(0)}, S_{t}^{(1)}\right)\right)_{t>0}$ be the $C_{0}$-semigroup in $\mathcal{F}\left(X_{0}, X_{1}\right)$ as in Proposition 3.8. Let $-B$ be the generator of $S^{\mathcal{F}}$. Then $D\left(A_{0}\right) \cap D\left(A_{1}\right) \subset D(B)$ and $D\left(A_{0}\right) \cap D\left(A_{1}\right)$ is a core for $B$.

Proof. Without loss of generality we may assume that both $S^{(0)}$ and $S^{(1)}$ are bounded semigroups. The resolvent

$$
(B+I)^{-1}: \mathcal{F}\left(X_{0}, X_{1}\right) \rightarrow D(B)
$$

is a topological isomorphism. Also the resolvent operators $(B+I)^{-1}$ and $\left(A_{0}+I\right)^{-1}$ are consistent by Proposition 3.8.(a) and Lemma 2.3. By Lemma 2.5](b) the restriction

$$
\left.(B+I)^{-1}\right|_{X_{0} \cap X_{1}}=\left.\left(A_{0}+I\right)^{-1}\right|_{X_{0} \cap X_{1}}: X_{0} \cap X_{1} \rightarrow D\left(A_{0}\right) \cap D\left(A_{1}\right)
$$


is a bijection. Because $X_{0} \cap X_{1} \subset \mathcal{F}\left(X_{0}, X_{1}\right)$, this implies immediately the assertion $D\left(A_{0}\right) \cap D\left(A_{1}\right) \subset D(B)$. Since $\mathcal{F}$ has Property $(\mathrm{d})$, the space $X_{0} \cap X_{1}$ is dense in $\mathcal{F}\left(X_{0}, X_{1}\right)$. Hence $D\left(A_{0}\right) \cap D\left(A_{1}\right)$ is dense in $D(B)$.

We provide the domain of a generator with the graph norm. Note that with the notation of the previous lemma, $\left(D\left(A_{0}\right), D\left(A_{1}\right)\right)$ is an interpolation couple and $A_{0} \in \mathcal{L}\left(D\left(A_{0}\right), X_{0}\right)$ and similarly $A_{1} \in \mathcal{L}\left(D\left(A_{1}\right), X_{1}\right)$. Now we are able to prove the main theorem of this paper.

Theorem 3.10. Let $\mathcal{F}$ be an interpolation functor which has Property (d). Let $\left(X_{0}, X_{1}\right)$ be an interpolation couple of Banach spaces. Further, let $S^{(0)}$ and $S^{(1)}$ be consistent $C_{0^{-}}$ semigroups in $X_{0}$ and $X_{1}$ with generators $-A_{0}$ and $-A_{1}$, respectively. Then $-\mathcal{F}\left(A_{0}, A_{1}\right)$ is the generator of the semigroup $\left(\mathcal{F}\left(S_{t}^{(0)}, S_{t}^{(1)}\right)\right)_{t>0}$.

In particular,

$$
D\left(\mathcal{F}\left(A_{0}, A_{1}\right)\right)=\mathcal{F}\left(D\left(A_{0}\right), D\left(A_{1}\right)\right) \text {. }
$$

Proof. Without loss of generality we may assume that $S^{(0)}$ and $S^{(1)}$ are bounded semigroups. Write $S_{t}^{\mathcal{F}}=\mathcal{F}\left(S_{t}^{(0)}, S_{t}^{(1)}\right)$ for all $t>0$ and let $-B$ be the generator of the $C_{0^{-}}$ semigroup $S^{\mathcal{F}}$. We know that $D\left(A_{0}\right) \cap D\left(A_{1}\right) \subset D(B)$ by Lemma 3.9. Also $B x=A_{0} x=$ $A^{\mathcal{F}} x$ for all $x \in D\left(A_{0}\right) \cap D\left(A_{1}\right)$ by Proposition 2.5](a), where we set $A^{\mathcal{F}}=\mathcal{F}\left(A_{0}, A_{1}\right)$. The operator $A^{\mathcal{F}}$ is bounded from $\mathcal{F}\left(D\left(A_{0}\right), D\left(A_{1}\right)\right)$ into $\mathcal{F}\left(X_{0}, X_{1}\right)$. Hence there exists a $c>0$ such that

$$
\left\|A^{\mathcal{F}} x\right\|_{\mathcal{F}\left(X_{0}, X_{1}\right)} \leq c\|x\|_{\mathcal{F}\left(D\left(A_{0}\right), D\left(A_{1}\right)\right)}
$$

for all $x \in \mathcal{F}\left(D\left(A_{0}\right), D\left(A_{1}\right)\right)$. If $x \in D\left(A_{0}\right) \cap D\left(A_{1}\right)$, then $B x=A^{\mathcal{F}} x$ and

$$
\|B x\|_{\mathcal{F}\left(X_{0}, X_{1}\right)} \leq c\|x\|_{\mathcal{F}\left(D\left(A_{0}\right), D\left(A_{1}\right)\right)} .
$$

Let $x \in \mathcal{F}\left(D\left(A_{0}\right), D\left(A_{1}\right)\right)$. Since $D\left(A_{0}\right) \cap D\left(A_{1}\right)$ is dense in $\mathcal{F}\left(D\left(A_{0}\right), D\left(A_{1}\right)\right)$ by Property (d), there exists a sequence $\left(x_{n}\right)_{n \in \mathbb{N}}$ in $D\left(A_{0}\right) \cap D\left(A_{1}\right)$ such that $\lim x_{n}=x$ in $\mathcal{F}\left(D\left(A_{0}\right), D\left(A_{1}\right)\right)$. Then $\left(B x_{n}\right)_{n \in \mathbb{N}}$ is a Cauchy sequence in $\mathcal{F}\left(X_{0}, X_{1}\right)$ and $\lim x_{n}=x$ in $\mathcal{F}\left(X_{0}, X_{1}\right)$. Since $B$ is a closed operator, it follows that $x \in D(B)$ and $B x=\lim B x_{n}=$ $\lim A^{\mathcal{F}} x_{n}=A^{\mathcal{F}} x$ in $\mathcal{F}\left(X_{0}, X_{1}\right)$. Hence $B$ is an extension of $A^{\mathcal{F}}$.

It remains to show that $D(B) \subset \mathcal{F}\left(D\left(A_{0}\right), D\left(A_{1}\right)\right)$. The operator $\left(A_{0}+I\right)^{-1}$ is bounded from $X_{0}$ into $D\left(A_{0}\right)$ and the operator $\left(A_{1}+I\right)^{-1}$ is bounded from $X_{1}$ into $D\left(A_{1}\right)$. Moreover, the operators $\left(A_{0}+I\right)^{-1}$ and $\left(A_{1}+I\right)^{-1}$ are consistent by Lemma 2.3, So by interpolation one obtains a bounded operator, denoted by $C$, from $\mathcal{F}\left(X_{0}, X_{1}\right)$ into $\mathcal{F}\left(D\left(A_{0}\right), D\left(A_{1}\right)\right)$. Let $c^{\prime}>0$ be such that

$$
\|C x\|_{\mathcal{F}\left(D\left(A_{0}\right), D\left(A_{1}\right)\right)} \leq c^{\prime}\|x\|_{\mathcal{F}\left(X_{0}, X_{1}\right)}
$$

for all $x \in \mathcal{F}\left(X_{0}, X_{1}\right)$. If $x \in X_{0} \cap X_{1}$, then $C x=\left(A_{0}+I\right)^{-1} x$. Hence

$$
\left\|\left(A_{0}+I\right)^{-1} x\right\|_{\mathcal{F}\left(D\left(A_{0}\right), D\left(A_{1}\right)\right)} \leq c^{\prime}\|x\|_{\mathcal{F}\left(X_{0}, X_{1}\right)}
$$

for all $x \in X_{0} \cap X_{1}$. Using Proposition 2.5 (b) it follows that

$$
\|x\|_{\mathcal{F}\left(D\left(A_{0}\right), D\left(A_{1}\right)\right)} \leq c^{\prime}\left\|\left(A_{0}+I\right) x\right\|_{\mathcal{F}\left(X_{0}, X_{1}\right)}=c^{\prime}\|(B+I) x\|_{\mathcal{F}\left(X_{0}, X_{1}\right)}
$$


for all $x \in D\left(A_{0}\right) \cap D\left(A_{1}\right)$. But $D\left(A_{0}\right) \cap D\left(A_{1}\right)$ is dense in $D(B)$ by Lemma 3.9. Since $\mathcal{F}\left(D\left(A_{0}\right), D\left(A_{1}\right)\right)$ is complete, it follows that $D(B) \subset \mathcal{F}\left(D\left(A_{0}\right), D\left(A_{1}\right)\right)$.

A similar statement is valid for the resolvents.

Proposition 3.11. Let $\mathcal{F}$ be an interpolation functor which has Property $(d)$. Let $\left(X_{0}, X_{1}\right)$ be an interpolation couple of Banach spaces. Further, let $S^{(0)}$ and $S^{(1)}$ be consistent bounded $C_{0}$-semigroups in $X_{0}$ and $X_{1}$ with generators $-A_{0}$ and $-A_{1}$, respectively. Then

$$
\mathcal{F}\left(\left(A_{0}+I\right)^{-1},\left(A_{1}+I\right)^{-1}\right)=\left(\mathcal{F}\left(A_{0}, A_{1}\right)+I\right)^{-1} .
$$

Proof. Write $S_{t}^{\mathcal{F}}=\mathcal{F}\left(S_{t}^{(0)}, S_{t}^{(1)}\right)$ for all $t>0$. Let $x \in X_{0} \cap X_{1}$. If $F \in\left(X_{0}+X_{1}\right)^{\prime}$, then

$$
\begin{aligned}
F\left(\left(\mathcal{F}\left(A_{0}, A_{1}\right)+I\right)^{-1} x\right) & =\int_{0}^{\infty} e^{-t} F\left(S_{t}^{\mathcal{F}} x\right) d t=\int_{0}^{\infty} e^{-t} F\left(S_{t}^{(0)} x\right) d t \\
& =F\left(\left(A_{0}+I\right)^{-1} x\right)=F\left(\mathcal{F}\left(\left(A_{0}+I\right)^{-1},\left(A_{1}+I\right)^{-1}\right) x\right) .
\end{aligned}
$$

So

$$
\left(\mathcal{F}\left(A_{0}, A_{1}\right)+I\right)^{-1} x=\mathcal{F}\left(\left(A_{0}+I\right)^{-1},\left(A_{1}+I\right)^{-1}\right) x .
$$

Moreover, the operator $\left(\mathcal{F}\left(A_{0}, A_{1}\right)+I\right)^{-1}$ is bounded from $\mathcal{F}\left(X_{0}, X_{1}\right)$ into itself. Hence $\mathcal{F}\left(\left(A_{0}+I\right)^{-1},\left(A_{1}+I\right)^{-1}\right)=\left(\mathcal{F}\left(A_{0}, A_{1}\right)+I\right)^{-1}$ by Lemma 3.7 .

\section{Example, $L^{p}$-spaces}

One of the commonly used theorems states that semigroups on $L^{p}$-spaces, which are induced by forms on $L^{2}$, extrapolate consistently to the whole $L^{p}$-scale, provided one knows Gaussian estimates for the $L^{2}$-semigroup. We next describe this situation.

Let $\Omega \subset \mathbb{R}^{d}$ be a bounded domain and $\mathcal{D} \subset \partial \Omega$ be closed. We define

$$
C_{\mathcal{D}}^{\infty}(\Omega)=\left\{\left.\psi\right|_{\Omega}: \psi \in C^{\infty}\left(\mathbb{R}^{d}\right) \text { and } \operatorname{supp} \psi \cap \mathcal{D}=\emptyset\right\}
$$

For all $p \in[1, \infty)$ let $W_{\mathcal{D}}^{1, p}(\Omega)$ be the closure of $C_{\mathcal{D}}^{\infty}(\Omega)$ in $W^{1, p}(\Omega)$. If $q \in(1, \infty]$, then we denote by $W_{\mathcal{D}}^{-1, q}(\Omega)$ the (anti-)dual of the space $W_{\mathcal{D}}^{1, q^{\prime}}(\Omega)$, where $q^{\prime}$ is the dual exponent of $q$. Let $\mu$ be a real, bounded, measurable, elliptic function on $\Omega$ which takes its values in the set of real $d \times d$-matrices. Define the sesquilinear form $\mathfrak{t}: W_{\mathcal{D}}^{1,2}(\Omega) \times W_{\mathcal{D}}^{1,2}(\Omega) \rightarrow \mathbb{C}$ by

$$
\mathfrak{t}[u, v]=\int_{\Omega} \mu \nabla u \cdot \overline{\nabla v}
$$

Let $A$ be the operator associated with $\mathfrak{t}$ in $L^{2}(\Omega)$ and let $\mathcal{A}: W_{\mathcal{D}}^{1,2}(\Omega) \rightarrow W_{\mathcal{D}}^{-1,2}(\Omega)$ be defined by $\langle\mathcal{A} u, v\rangle=\mathfrak{t}[u, v]$ for all $u, v \in W_{\mathcal{D}}^{1,2}(\Omega)$. Then $A$ and $\mathcal{A}$ generate analytic semigroups $S^{(2)}$ and $\widetilde{S}^{(2)}$ on $L^{2}(\Omega)$ and $W_{\mathcal{D}}^{-1,2}(\Omega)$, respectively.

Theorem 4.1. Adopt the above notation and assumptions. 
(a) The semigroups $S^{(2)}$ and $\widetilde{S}^{(2)}$ are consistent.

(b) If the boundary around any point $x \in \overline{\partial \Omega \backslash \mathcal{D}}$ admits a bi-Lipschitzian boundary chart, then the semigroup $S^{(2)}$ on $L^{2}(\Omega)$ has a kernel with Gaussian upper estimates. Moreover, the semigroup $S^{(2)}$ extends consistently to a $C_{0}$-semigroup $S^{(p)}$ on $L^{p}(\Omega)$ for all $p \in[1, \infty)$.

Proof. (a). See Ouh Subsection 1.4.2.

(b)]. The first assertion is proved in ER] Theorem 3.1. The second one follows from the first by Are second proof on page 1160 .

It is desirable in various contexts to know the consistency of semigroups on spaces like $L^{p}(\Omega)$ and $W_{\mathcal{D}}^{-1, q}(\Omega)$ - as outlined in the introduction. Before we prove such a result we establish the following lemma.

Lemma 4.2. Let $p \in[1, \infty)$ and $q \in(1, \infty)$. Then $C_{c}^{\infty}(\Omega)$ is dense in $W_{\mathcal{D}}^{-1, q}(\Omega) \cap L^{p}(\Omega)$.

Proof. First of all, $W_{\mathcal{D}}^{-1, q}(\Omega) \cap L^{p}(\Omega)$ is dense in both $W_{\mathcal{D}}^{-1, q}(\Omega)$ and $L^{p}(\Omega)$, since $C_{c}^{\infty}(\Omega) \subset$ $W_{\mathcal{D}}^{-1, q}(\Omega) \cap L^{p}(\Omega)$. Therefore

$$
\left(W_{\mathcal{D}}^{-1, q}(\Omega) \cap L^{p}(\Omega)\right)^{\prime}=\left(W_{\mathcal{D}}^{-1, q}(\Omega)\right)^{\prime}+\left(L^{p}(\Omega)\right)^{\prime}=W_{\mathcal{D}}^{1, q^{\prime}}(\Omega)+L^{p^{\prime}}(\Omega),
$$

cf. [BL76] Theorem 2.7.1. Let $F \in\left(W_{\mathcal{D}}^{-1, q}(\Omega) \cap L^{p}(\Omega)\right)^{\prime}=W_{\mathcal{D}}^{1, q^{\prime}}(\Omega)+L^{p^{\prime}}(\Omega)$ and suppose that $F(u)=0$ for all $u \in C_{c}^{\infty}(\Omega)$. Then $F \in L^{1}(\Omega)$. So $F=0$. Then the statement follows from the Hahn-Banach theorem.

For all $p \in[1, \infty)$ let $S^{(p)}$ be the semigroup on $L^{p}(\Omega)$ as in Theorem 4.1](b) (assuming the conditions of that theorem are satisfied).

Theorem 4.3. Assume that the boundary around any point $x \in \overline{\partial \Omega \backslash \mathcal{D}}$ admits a biLipschitzian boundary chart. Then the semigroup $\widetilde{S}^{(2)}$ is consistent with the semigroup $S^{(p)}$ for every $p \in[1, \infty)$.

Proof. Let $t>0$. If $u \in C_{c}^{\infty}(\Omega)$, then $\widetilde{S}_{t}^{(2)} u=S_{t}^{(2)} u=S_{t}^{(p)} u$ by Theorem 4.1. Now the result follows from Lemmas 2.2 and 4.2 .

There is also a version for $W_{\mathcal{D}}^{-1, q}(\Omega)$ with $q \in[2, \infty)$ under slightly more assumptions.

\section{Theorem 4.4. Suppose that}

- the boundary around any point $x \in \overline{\partial \Omega \backslash \mathcal{D}}$ admits a bi-Lipschitzian boundary chart,

- the set $\mathcal{D}$ is a $(d-1)$-set in the sense of Jonsson-Wallin [JW] Chapter II and

- $\Omega$ is a d-set in the sense of Jonsson-Wallin, or for all $x \in \Omega$ the matrix $\mu(x)$ is symmetric. 
Let $q \in[2, \infty)$. Define the operator $\widetilde{\mathcal{A}}_{q}$ in $W_{\mathcal{D}}^{-1, q}(\Omega)$ by

$$
D\left(\widetilde{\mathcal{A}}_{q}\right)=\left\{\psi \in W_{\mathcal{D}}^{-1, q}(\Omega) \cap W_{\mathcal{D}}^{1,2}(\Omega): \mathcal{A} \psi \in W_{\mathcal{D}}^{-1, q}(\Omega)\right\}
$$

and $\widetilde{\mathcal{A}}_{q}=\left.\mathcal{A}\right|_{D\left(\widetilde{\mathcal{A}}_{q}\right)}$. Then $-\widetilde{\mathcal{A}}_{q}$ generates a holomorphic semigroup on $W_{\mathcal{D}}^{-1, q}(\Omega)$ which is consistent with the semigroup $S^{(p)}$ for all $p \in[1, \infty)$.

Proof. It follows from DER] Lemma $6.9(\mathrm{c})$ that $-\widetilde{\mathcal{A}}_{q}$ generates a holomorphic semigroup on $W_{\mathcal{D}}^{-1, q}(\Omega)$. Denote this semigroup by $\widetilde{S}^{(q)}$. Then $\widetilde{S}^{(q)}$ is consistent with $\widetilde{S}^{(2)}$ by the paragraph before Lemma 6.9 in [DER]. Hence if $t>0$ and $u \in C_{c}^{\infty}(\Omega)$, then $\widetilde{S}_{t}^{(q)} u=$ $\widetilde{S}_{t}^{(2)} u=S_{t}^{(p)} u$. Finally use again Lemmas 2.2 and 4.2 ,

\section{Acknowledgements}

Part of this work is supported by the Marsden Fund Council from Government funding, administered by the Royal Society of New Zealand.

\section{References}

[Ama] Amann, H., Dual semigroups and second order linear elliptic boundary value problems. Isreal J. Math. 45 (1983), 225-254.

[AE] Amann, H. and Escher, J., Strongly continuous dual semigroups. Ann. Mat. Pura Appl. (4) 171 (1996), 41-62.

[Are] Arendt, W., Gaussian estimates and interpolation of the spectrum in $L^{p}$. Diff. Int. Equ. 7 (1994), 1153-1168.

[BL76] Bergh, J. and Löfström, J., Interpolation spaces. An introduction. Grundlehren der mathematischen Wissenschaften 223. Springer-Verlag, Berlin etc., 1976.

[BMnR] Bonifacius, L., Meinlschmidt, H., Neitzel, I. and Rehberg, J., Global solutions for quasilinear parabolic equations in a scale of Banach spaces, 2017. In preparation.

[BB] Butzer, P. L. and Berens, H., Semi-groups of operators and approximation. Die Grundlehren der mathematischen Wissenschaften 145. SpringerVerlag, Berlin etc., 1967.

[Cia] Ciarlet, P. G., The finite element method for elliptic problems. Studies in Mathematics and its Applications 4. North-Holland, Amsterdam, 1978.

[CS] Clément, P. and Simonett, G., Maximal regularity in continuous interpolation spaces and quasilinear parabolic equations. J. Evol. Equ. 1 (2001), 39-67. 
[DER] Disser, K., Elst, A. F. M. ter and Rehberg, J., On maximal parabolic regularity for non-autonomous parabolic operators. J. Differential Equations 262 (2017), 2039-2072.

[ER] Elst, A. F. M. Ter and RehberG, J., $L^{\infty}$-estimates for divergence operators on bad domains. Anal. and Appl. 10 (2012), 207-214.

[GGZ] Gajewski, H., GröGer, K. and Zacharias, K., Nichtlineare Operatorgleichungen und Operatordifferentialgleichungen. Mathematische Lehrbücher und Monographien, II. Abteilung Mathematische Monographien 38. AkademieVerlag, Berlin, 1974.

[GGKR] Griepentrog, J. A., Gröger, K., Kaiser, H.-C. and Rehberg, J., Interpolation for function spaces related to mixed boundary value problems. Math. Nachr. 241 (2002), 110-120.

[HDR] Haller-Dintelmann, R. and Rehberg, J., Maximal parabolic regularity for divergence operators including mixed boundary conditions. J. Differential Equations 247 (2009), 1354-1396.

[HhkrZ] Haller-Dintelmann, R., Höppner, W., Kaiser, H.-C., Rehberg, J. and Ziegler, G. M., Optimal elliptic regularity in Sobolev spaces near threedimensional multimaterial Neumann vertices. Funct. Anal. Appl. 48 (2014), $208-222$.

[HR] Hieber, M. and Rehberg, J., Quasilinear parabolic systems with mixed boundary conditions on nonsmooth domains. SIAM J. Math. Anal. 40 (2008), $292-305$.

[JW] Jonsson, A. and Wallin, H., Function spaces on subsets of $\mathbf{R}^{n}$. Math. Rep. 2, No. 1 (1984).

[Lio] Lions, J. L., Contrôle optimal de systèmes gouvernés par des équations aux dérivées partielles. Dunod/Gauthier-Villars, Paris, 1968.

[Lun] LUnARDI, A., Analytic semigroups and optimal regularity in parabolic problems, vol. 16 of Progress in Nonlinear Differential Equations and their Applications. Birkhäuser Verlag, Basel, 1995.

[MerS] Maz'ya, V., Elschner, J., Rehberg, J. and Schmidt, G., Solutions for quasilinear nonsmooth evolution systems in $L^{p}$. Arch. Ration. Mech. Anal. 171 (2004), 219-262.

[Ouh] Ouhabaz, E.-M., Analysis of heat equations on domains, vol. 31 of London Mathematical Society Monographs Series. Princeton University Press, Princeton, NJ, 2005. 
[Tri] Triebel, H., Interpolation theory, function spaces, differential operators. North-Holland, Amsterdam, 1978.

[Zan] Zanger, D., The inhomogeneous Neumann problem in Lipschitz domains. Comm. Partial Differential Equations 25 (2000), 1771-1808.

A.F.M. ter Elst, Department of Mathematics, University of Auckland, Private Bag 92019, Auckland 1142, New Zealand

E-mail address: terelst@math.auckland.ac.nz

J. Rehberg, Weierstrass Institute for Applied Analysis and Stochastics, MohrenStr. 39, 10117 Berlin, Germany

E-mail address: rehberg@wias-berlin.de 